\title{
EMBEDDING A TRANSFORMATION GROUP IN AN AUTOMORPHISM GROUP'
}

\author{
MURRAY EISENBERG
}

1. Introduction. Using a construction of Baĭdosov [1], we show that a topological transformation group with completely regular phase space $X$ and locally compact phase group $T$ can be equivariantly embedded in a transformation group of automorphisms of a topological group $A$. The group $A$ in question is the free abelian topological group over $X$; some facts about $A$ are established in $\S 2$. In $\$ 4$ several dynamical properties of $(A, T)$ are discussed as they relate to properties of $(X, T)$.

As general references to the notation and notions for transformation groups used here, see [4] and [5].

All topological spaces considered below, and in particular all topological groups, are assumed to be Hausdorff.

2. The topological group $A(X)$. Let $X$ be a completely regular space. Denote by $A(X)$ or simply $A$ the free abelian topological group over $X[\mathbf{6}],[\mathbf{7}, \S 8]$. Algebraically $A$ is just the free abelian group generated by the set $X$; the topology of $A$ is the greatest separated topology compatible with the group structure and inducing on $X$ a topology weaker than the one initially given on $X$. We have:

(1) $A$ is a topological group containing $X$ as a closed subspace.

(2) If $f$ is a continuous map of $X$ into an abelian topological group $G$, then the unique extension of $f$ to a group homomorphism of $A$ into $G$ is continuous.

Proposition 1. Let $X$ be compact and infinite. Then $A$ is not a Baire space and a fortiori is not locally compact.

Proof. If $z \in A$ and $z \neq 0$, there exist distinct $x_{1}, \cdots, x_{n} \in X$ and nonzero integers $\alpha_{1}, \cdots, \alpha_{n}$ with $z=\sum_{i} \alpha_{i} x_{i}$, and we let $L(z)$ $=\sum_{i}\left|\alpha_{i}\right|$. Set $L(0)=0$. For each positive integer $n$ let $A_{n}$ $=\{z \mid z \in A, L(z) \leqq n\}$. Since $A=\mathrm{U}_{1}^{\infty} A_{n}$, it is enough to show that each $A_{n}$ is compact and has vacuous interior.

Let $n>0$. Let $B_{0}=\{0\}$, and for each positive integer $p$ let $B_{p}$ be the set of all elements of $A$ of the form $\sum_{1}^{p} x_{i}$ where $x_{1}, \cdots, x_{p} \in X$, not necessarily distinct. Then $A_{n}=\cup\left\{B_{p}-B_{q} \mid 0 \leqq p+q \leqq n\right\}$, where

Received by the editors August 29, 1968.

1 This work was partially supported by National Science Foundation grants GP5972 and GP-6651. 
$B_{p}-B_{q}$ denotes the algebraic difference, so $A_{n}$ is compact.

Suppose $A_{n}$ contains a nonempty open subset $W$ of $A$. Choose $v \in W$ and let $U=-v+W$. Since $A$ is nondiscrete, there exists $z \in A$ with $z \neq 0$ and $(2 n+1) z \in U$. Let $u=(2 n+1) z$. Then $L(u)$ $=(2 n+1) L(z) \geqq 2 n+1$. On the other hand, $u=-v+w$ for some $w \in W$, whence $L(u) \leqq L(v)+L(w) \leqq 2 n$.

Proposition 2. Let $\phi: X \rightarrow Y$ be a continuous surjection of compact spaces. Then the canonical map $\phi^{*}: A(X) \rightarrow A(Y)$ induced by $\phi$ is a continuous-open group epimorphism.

Proof. Let $N=\operatorname{ker} \phi^{*}, G=A(X) / N$. The map $\psi: G \rightarrow A(Y)$ associated with $\phi^{*}$ is continuous and is an algebraic isomorphism. To show that $\phi^{*}$ is open, we prove that $\psi$ is homeomorphic.

Let $B=Y \psi^{-1}$, and let $B_{1}$ be the image of $X$ under the projection of $A(X)$ onto $G$, so that $B_{1} \subset B$. Now $B_{1}$ generates $G$ since $X$ generates $A(X)$, and $B$ is free in $G$ since $Y$ is free in $A(Y)$. Then $B_{1}=B$. Since $B$ is closed in $G$, it follows that $G=A(B)$. Moreover, $\psi$ maps $B$ homeomorphically onto $Y$, so $\psi$ is the canonical map of $A(B)$ into $A(Y)$ induced by $\psi \mid B$. Hence $\psi$ is homeomorphic.

3. The transformation group $(A(X), T)$. Now let the completely regular space $X$ be the phase space of a transformation group ( $X$, $T, \pi)$. Following Baĭdosov [1], we extend $\pi$ to an action $\pi^{*}$ of $T$ on $A=A(X)$ as follows. Let $j$ be the inclusion map of $X$ into $A$. For each $t \in T$ let $\pi^{t^{*}}$ be the continuous endomorphism of $A$ which extends the continuous map $\pi^{t} j$, where $\pi^{t}$ is the $t$-transition given by $x \pi^{t}=(x, t) \pi$ for $x \in X$. For $z \in A$ and $t \in T$ let $(z, t) \pi^{*}=z \pi^{t^{*}}$. We call $\pi^{*}$ the free extension of $\pi$.

If $\phi$ is a homomorphism of $(X, T, \pi)$ into another transformation group $(Y, T, \sigma)$, with $Y$ completely regular, evidently the map $\phi^{*}: A(X) \rightarrow A(Y)$ induced by $\phi$ is equivariant with respect to $\pi^{*}$ and $\sigma^{*}$.

Theorem 1. Suppose $T$ is locally compact. Then $\pi^{*}$ is continuous, $\left(A, T, \pi^{*}\right)$ is a transformation group whose transitions are automorphisms of $A$, and $j$ is an isomorphism of $(X, T, \pi)$ into $\left(A, T, \pi^{*}\right)$.

Proof. The only nontrivial fact to be proved is the continuity of $\pi^{*}$. Denote by $C(T, X), C(T, A)$ the sets of all continuous maps of $T$ into $X, A$ respectively, and endow these function spaces with their compact-open topologies. Define $\mu^{*}: A \rightarrow A^{T}$ by $t\left(z \mu^{*}\right)=(z, t) \pi^{*}$ for $t \in T, z \in A$. Since $T$ is locally compact, it suffices to show that $\mu^{*}$ maps $A$ continuously in to $C(T, A)$. 
We show that $A \mu^{*} C C(T, A)$. If $x \in X$, then $t \in T$ implies $t\left(x \mu^{*}\right)$ $=(x, t) \pi$, so $x \mu^{*} \in C(T, A)$ by continuity of $\pi$. Now let $z \in A$. Choose $x_{1}, \cdots, x_{n} \in X$ and integers $\alpha_{1}, \cdots, \alpha_{n}$ with $z=\sum \alpha_{i} x_{i}$. Then $z \mu^{*}=\sum \alpha_{i}\left(x_{i} \mu^{*}\right)$, and $z \mu^{*} \in C(T, A)$.

Let $j^{*}$ be the canonical injection of $C(T, X)$ into $C(T, A)$, so that $j^{*}$ is continuous. Define $\mu: X \rightarrow X^{T}$ by $t(x \mu)=(x, t) \pi$ for $t \in T, x \in X$. Then $\mu$ is a continuous map of $X$ into $C(T, X)$, so $\psi=\mu j^{*}$ is a continuous map of $X$ into $C(T, A)$.

The addition on $C(T, A)$ defined pointwise makes this space into a separated abelian group. Hence $\psi$ extends to a continuous homomorphism $\psi^{*}$ of $A$ in to $C(T, A)$. But $\mu^{*}$ is also a group homomorphism of $A$ into $C(T, A)$ extending $\psi$. It follows that $\mu^{*}=\psi^{*}$, and $\mu^{*}$ is continuous.

Corollary. Suppose $X$ is compact and $(X, T, \pi)$ is equicontinuous. Then $\pi^{*}$ is continuous.

Proof. Let $E$ be the enveloping semigroup [4] of $(X, T, \pi)$. Then $E$ is a compact group of homeomorphisms of $X$ onto $X$, and the evaluation map $\sigma: X \times E \rightarrow X$ is continuous and defines a transformation group $(X, E, \sigma)$. By the theorem the free extension $\sigma^{*}$ of $\sigma$ is continuous. The map $\mu: T \rightarrow E$ such that $t \in T$ implies $t \mu=\pi^{t}$ is continuous. The continuity of $\pi^{*}$ now follows from the factorization $\pi^{*}=(i \times \mu) \sigma^{*}$, where $i$ is the identity map of $X$.

REMARK. Instead of the free abelian topological group $A$ over $X$, consider the free linear topological space $V$ over $X$ [9]. If $T$ is locally compact, then one can still show as above that $\pi$ has a continuous extension $\pi^{*}: V \times T \rightarrow V$ making $\left(V, T, \pi^{*}\right)$ a transformation group of linear automorphisms of $V$.

4. Dynamical properties of $(A(X), T)$. Again $(X, T, \pi)$ denotes a transformation group, where $X$ is completely regular. Continuity of $\pi^{*}$ is not needed below, and we suppress explicit mention of $\pi$ and $\pi^{*}$.

THEOREM 2. The action of $T$ on $A$ is not topologically ergodic, that $i s$, there exist nonempty open subsets $N$ and $M$ of $A$ with $N t \cap M=\varnothing$ for all $t \in T$.

Proof. Let $D_{0}$ be the constant map on $X$ with range $\{1\}$, let $D$ be the homomorphism of $A$ into the additive group of integers which extends $D_{0}$, and let $N=\operatorname{ker} D$. Then $N$ is a $T$-invariant subgroup of $A$, so it is enough to show that $N$ is open in $A$.

Let $J_{0}, \Im$ be the topologies of $X, A$ respectively. The topology $s$ of $A$ generated by $\{N\}$ and $I$ is separated and compatible with the 
group structure of $A$. Let $\mathcal{S}_{0}$ be the topology on $X$ induced by $\mathrm{S}$. It is now enough to show $S_{0} \subset J_{0}$, for then $N \in S \subset J$.

Let $z \in A$ and $V \in J$ with $(z+N) \cap V \cap X \neq \varnothing$. It remains to show $(z+N) \cap V \cap X \in 3_{0}$. Choose any $y \in(z+N) \cap X$. If $x \in X$, then $x=y+(x-y) \in z+N+N=z+N$. Hence $X \subset z+N$, and $(z+N) \cap V$ $\cap X=V \cap X \in J_{0}$.

In case $X$ is connected, the group $N$ in the preceding proof is just the identity component of $A$.

We use below a theorem of Ellis [3] stating that if $T$ acts as a homeomorphism group of a space $Y$ in which each orbit is relatively compact, then $(Y, T)$ is distal if and only if $\left(Y^{n}, T\right)$ is pointwise almost periodic for some $n>1$, or equivalently, $\left(Y^{n}, T\right)$ is pointwise almost periodic for every $n>0$. This applies to $(A, T)$ when $X$ is compact, because $A$ is the union of the compact sets $A_{n}$ constructed in the proof of Proposition 1, and each $A_{n}$ is $T$-invariant.

To accommodate thecommutativity of addition in $A$, it isconvenient to use the symmetric product $X * X$ of $X$ with itself [2]. Here $X * X$ is the compact space obtained by identifying each $(x, y) \in X \times X$ with $(y, x)$. Let $p: X \times X \rightarrow X * X$ be the projection, and for $(x, y)$ $\in X \times X$ let $x * y$ denote $(x, y) p$. The map $(x * y, t) \mapsto x t * y t$ of $X \times T$ into $X * X$ is well defined and makes $(X * X, T)$ a transformation group and $p$ a homomorphism.

THEOREM 3. The following statements are equivalent when $X$ is compact:

(1) $(X, T)$ is distal.

(2) $(A, T)$ is distal.

(3) $(A, T)$ is pointwise almost periodic.

Proof. Assume (1). We show (2). Let $z \in A$ with $z \neq 0$. It is enough to show that $z$ is distal from 0 . The compact $T$-invariant set $A_{1}=-X$ $\cup X \cup\{0\}$ is distal under $T$, so $\left(A_{1}^{n}, T\right)$ is distal for each $n$. But for sufficiently large $n$ both 0 and $z$ belong to the range of the homomorphism $\left(z_{1}, \cdots, z_{n}\right) \mapsto \sum z_{i}$ of $\left(A_{1}^{n}, T\right)$ into $(A, T)$.

By Ellis' theorem, (2) implies (3).

Assume (3). We show (1). We have an obvious isomorphism of $(X * X, T)$ with $(X+X, T)$, so $(X * X, T)$ is pointwise almost periodic. Let $x, y \in X$ with $x \neq y$. Choose disjoint compact neighborhoods $U, V$ of $x, y$ in $X$. Then $(U \times V) p$ is a neighborhood of $x * y$ in $X * X$, and $(x * y) T \subset(U \times V) p K$ for some compact subset $K$ of $T$. Then

$$
\alpha=(X \times X) \backslash((U \times V) \cup(V \times U)) K
$$


is an index of the uniformity of $X$, and $(x, y) T$ is disjoint from $\alpha$.

The following lemma concerning lifting of minimality is of interest in its own right (cf. [0, p. 27], [8, 2.1]).

Lemma. Let $(X, T),(Y, T)$ be transformation groups, where $X, Y$ are compact, and let $\phi$ be a locally one-to-one homomorphism of $(X, T)$ onto $(Y, T)$. Suppose $(Y, T)$ is minimal and $(X, T)$ has a dense orbit. Then $(X, T)$ is minimal.

Proof. Suppose $(X, T)$ is not minimal. Choose $x_{0} \in X$ with $x_{0} T$ dense in $X$, and set $y_{0}=x_{0} \phi$. There exists some minimal subset $M$ of $X$. The fiber $y_{0} \phi^{-1}$ over $y_{0}$ is finite and, since $\phi$ maps $M$ onto $Y$, meets $M$. Let $y_{0} \phi^{-1}=\left\{x_{0}, x_{1}, \cdots, x_{n}\right\}$ with $y_{0} \phi^{-1} \cap M=\left\{x_{m}\right.$, $\left.x_{m+1}, \cdots, x_{n}\right\}$.

Choose pairwise disjoint open neighborhoods $W_{0}, \cdots, W_{n}$ of $x_{0}, \cdots, x_{n}$ with $\phi$ one-to-one on each $W_{i}$ and with $M$ disjoint from the closure of $W_{i}$ for $0<i<m$. For each $z \notin \mathrm{U}_{0}^{n} W_{i}$ there exist disjoint neighborhoods of $y_{0} \phi^{-1}$ and $z \phi \phi^{-1}$ which are saturated by $\phi$. A standard compactness argument produces a saturated neighborhood $U$ of $y_{0} \phi^{-1}$ such that $U \subset \bigcup_{0}^{n} W_{i}$. For $i=0, \cdots, n$ let $U_{i}=U \cap W_{i}$, and let $V=U \phi$, whence $V$ is a neighborhood of $y_{0}$.

Because $(Y, T)$ is minimal it is discretely almost periodic at $y_{0}$, and there exist subsets $S, K$ of $T$ with $K$ finite, $T=S K$, and $y_{0} S \subset V$. Then $x_{i} S \subset U$ for each $i$.

There exists a net $\left(s_{j}, k_{j}\right)_{j}$ in $S \times K$ such that $\lim x_{0} s_{j} k_{j}=x_{m}$. By passing to a subnet if necessary, we may assume $k_{j}=k$ for some $k$ and all $j$. Then $\lim x_{0} s_{j}=x_{m} k^{-1} \in M$, so $x_{0} S \nsubseteq \cup_{0}^{m-1} U_{i}$, and $x_{0} \in \in U_{p}$ for some $s \in S$ and some $p \geqq m$. It follows that some two of the $n-m+2$ points $x_{0} s, x_{m} s, \cdots, x_{n} s$ belong to the same one of the $n-m+1$ sets $U_{m}, \cdots, U_{n}$. This is impossible since $x_{i} s \phi=y_{0} s$ for all $i$ and $\phi$ is one-to-one on each $U_{i}$.

THEOREM 4. Let $x, y \in X$ with $x \neq y$. Then $(x, y)$ is almost periodic under $(X \times X, T)$ if and only if $x+y$ is almost periodic under $(A, T)$.

Proof. The restriction to $X \times X$ of addition in $A$ is a homomorphism of $(X \times X, T)$ into $(A, T)$. Hence $x+y$ is almost periodic if $(x, y)$ is.

Conversely, assume $x+y$ is almost periodic. We first show that $x$ is distal from $y$ in $X$. Choose a symmetric index $\alpha$ of the uniformity of $X$ with $(x, y) \notin \alpha^{3}$. Since $x * y$ is almost periodic under $(X * X, T)$, the set

$$
S=\{s \mid s \in T,(x s, y s) \in(x \alpha \times y \alpha) \cup(y \alpha \times x \alpha)\}
$$


is left syndetic in $T$, and $T=S K$ for some compact set $K$. Choose an index $\beta$ of $X$ with $\beta K^{-1} \subset \alpha$. Then $(x, y) T \cap \beta=\varnothing$.

Since $x$ is distal from $y$, the orbit-closure $B$ of $(x, y)$ in $X \times X$ is disjoint from the diagonal of $X \times X$. Then $p \mid B$ is a locally one-to-one (in fact, locally homeomorphic) homomorphism of $B$ onto the orbitclosure of $x * y$ in $X * X$. The almost periodicity of $(x, y)$ now follows from that of $x * y$ by means of the lemma.

A similar but more direct argument shows that $(x, y)$ is almost periodic if and only if $x-y$ is.

ADDED IN PROOF. Mr. Leonard Shapiro has kindly pointed out that a proposition equivalent to our lemma appears in a paper of $\mathrm{R}$. Ellis [Amer. J. Math 87 (1965), 564-574]. Ellis' proof is entirely different from ours in that it employs the enveloping semigroup of a transformation group.

\section{REFERENCES}

0. L. Auslander, L. Green and F. Hahn, Flows on homogeneous spaces, Princeton Univ. Press, Princeton, N. J., 1963.

1. V. A. BaǏdosov, Invariant functions of dynamical systems, Izv. Vysš. Učebn. Zaved. Matematika 1959, no. 1 (8), 9-15. (Russian)

2. K. Borsuk and S. Ulam, On symmetric products of topological spaces, Bull. Amer. Math. Soc. 37 (1931), 875-882.

3. Robert Ellis, Distal transformation groups, Pacific J. Math. 8 (1958), 401-405.

4. Robert Ellis and W. H. Gottschalk, Homomorphisms of transformation groups, Trans. Amer. Math. Soc. 94 (1960), 258-271.

5. W. H. Gottschalk and G. A. Hedlund, Topological dynamics, Amer. Math. Soc. Colloq. Publ., vol. 36, Amer. Math. Soc., Providence, R. I., 1955.

6. M. I. Graev, Theory of topological groups. I: Norms and metrics on groups. Complete groups. Free topological groups, Uspehi Mat. Nauk 5 (1950), no. 2 (36), 6-56. (Russian)

7. E. Hewitt and K. A. Ross, Abstract harmonic analysis. I, Academic Press, New York, 1963.

8. P. J. Kahn and A. W. Knapp, Equivariant maps onto minimal flows, Math. Systems Theory 2 (1968), 319-324.

9. S. Kakutani, Free topological groups and infinite direct product topological groups, Proc. Imp. Acad. Tokyo 20 (1944), 595-598.

University of Massachusetts, Amherst 